\title{
Erroneous high ventricular rate (HVR) diagnosis with the BioMonitor 2-AF implantable cardiac monitor due to P-wave oversensing - case report and programming solutions
}

\author{
Damian Małecki ${ }^{1, \mathrm{~B}-\mathrm{D}, \mathrm{F}}$, Łukasz Januszkiewicz ${ }^{1, \mathrm{C}-\mathrm{E}}$, Marcin Michalak $^{1, \mathrm{C}-\mathrm{E}}$, Marcin Grabowski ${ }^{1, \mathrm{~A}, \mathrm{E}-\mathrm{F}}$ \\ A - Research concept and design, B - Collection and/or assembly of data, C - Data analysis and interpretation, \\ D - Writing the article, E - Critical revision of the article, F - Final approval of article \\ 1. $1^{\text {st }}$ Department of Cardiology, Medical University of Warsaw
}

Address for correspondence:

Damian Małecki, $1^{\text {st }}$ Department of Cardiology, Medical University of Warsaw email: dammalecki@gmail.com

Łukasz Januszkiewicz, $1^{\text {st }}$ Department of Cardiology, Medical University of Warsaw email: lukasz.jan.januszkiewicz@gmail.com

Marcin Michalak, $1^{\text {st }}$ Department of Cardiology, Medical University of Warsaw email: mmajkelak@gmail.com

Marcin Grabowski, $1^{\text {st }}$ Department of Cardiology, Medical University of Warsaw email: marcin.grabowski@wum.edu.pl

Received: 12.01 .2018

Revised: 22.01.2018

Accepted: 11.02.2018

Final review: 23.01.2018

DOI: $10.24255 / \mathrm{hbj} / 85213$

\section{Key words:}

\section{biomonitor, telemonitoring, implantable cardiac monitor, oversensing, PWOS}

\begin{abstract}
Implantable cardiac monitors (ICMs) are valuable tools for diagnosis of syncope and arrhythmia. However, ICMs may be responsible for a significant number of misclassified records due to artefacts and/or imperfections of diagnostic algorithms. $\mathrm{P}$-wave oversensing (PWOS) is one of the plausible causes of inappropriate automatic analysis. In this report, a case of a free diver with recurrent syncope is investigated. The patient had
\end{abstract}

\section{Introduction}

An implantable cardiac monitor (ICM) is a valuable tool for diagnosis of various disorders including unexplained syncope, palpitations, suspected bradyarrhythmia and tachyarrhythmia ${ }^{[1,2]}$. The device is implanted subcutaneously in the precordial area with battery longevity up to three years and has much higher effectiveness than classic diagnostic methods such as a single electrocardiogram (ECG) and shortor long-term Holter monitoring, especially in cases of rare episodes of syncope or arrhythmia. Thus, implantation of an ICM is recommended by the European Society of Cardiology in selected patients with recurrent unexplained syncope ${ }^{[3]}$.

While being undoubtedly useful, ICMs are responsible for a significant number of incorrectly analysed impulses, mostly undergone the implantation of a Biomonitor 2-AF of Biotronik $\mathrm{GmbH}$. Soon after implantation, high ventricular rhythm (HVR) was registered. Manual analysis of the incident report revealed mild bradycardia and repetitive $\mathrm{P}$-wave and periodic T-wave oversensing misclassified as HVR. The mechanism of PWOS and management options are discussed.

due to oversensing. This may lead to misclassification of sinus rhythm as ventricular arrhythmia or atrial fibrillation ${ }^{[1]}$.

\section{Case report}

A 44-year-old man was referred to the clinic for implantation of an ICM due to multiple episodes of syncope. The patient was a free diver (breath-hold diver) and wanted to continue his activities. Previous diagnostic tests revealed sinus bradycardia and type 1 second-degree atrioventricular block at night without significant pauses. Due to the high risk of a fatal clinical course (one of the syncopal episodes occurred during diving), implantation of a dual-chamber pacemaker was suggested, but the patient refused the treatment at that time. 
The implantable cardiac monitor BioMonitor 2-AF (Biotronik $\mathrm{GmbH}$, Germany) was implanted and the patient was enrolled in the HomeMonitoring telemonitoring system. A month after implantation an arrhythmic event classified as high ventricular rate (HVR) was registered and transferred to the Telemonitoring Centre. The incident occurred in the morning and lasted 12 seconds. Analysis of the report made by a physician revealed mild sinus bradycardia of $50 \mathrm{bpm}$ misinterpreted as HVR due to repetitive $\mathrm{P}$-wave and periodic T-wave oversensing (Fig. 1).

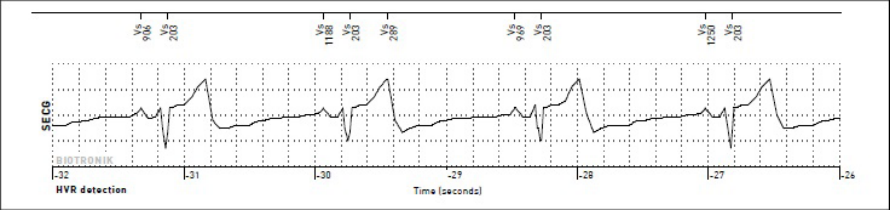

Figure 1.

\section{Discussion}

An implantable cardiac monitor is an invaluable tool to diagnose infrequent arrhythmias that cannot be captured with traditional, less invasive methods, such as the resting 12lead electrocardiogram, Holter monitoring or external event recorders. If functioning properly, the device can lead to fast and precise diagnosis of the patient's condition and therefore to earlier initiation of proper treatment ${ }^{[4]}$. However, like every diagnostic tool based on a surface electrocardiogram, ICMs are prone to report a significant number of erroneous records due to artefacts and/or imperfections of diagnostic algorithms. One of the possible causes of inappropriate automatic diagnosis is P-wave oversensing (PWOS).

The PWOS phenomenon occurs when the device - which normally detects only R-waves in order to examine ventricular rhythm - detects a P-wave and interprets it as a ventricular impulse. Consequent $\mathrm{P}$-wave oversensing may lead to incorrect classification of sinus rhythm as HVR. However, as can be seen in the tachogram and Lorenz plot, the Vs-Vs intervals vary significantly in time (Fig. 2). This would not occur in correctly

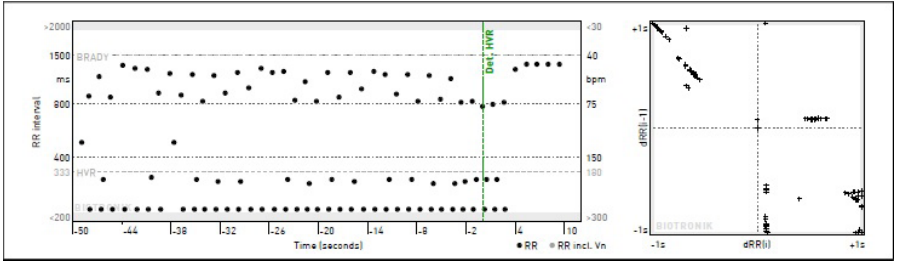

Figure 2.

identified HVR, when we would see a more regular length of $\mathrm{V}-\mathrm{V}$ intervals. The short purported $\mathrm{V}-\mathrm{V}$ intervals are in fact PR intervals (or, less often, intervals between the R-wave and oversensed T-wave), while long V-V intervals correspond to intervals between the R-wave and oversensed subsequent $\mathrm{P}$-wave. The P-wave will be detected if its amplitude exceeds the target sensing threshold nominally set to a $25 \%$ limit of the maximum sensed R-wave amplitude ${ }^{[1]}$.

There are some actions available to prevent PWOS (and TWOS). First, the sensing high pass filter can be set to higher values, from the default $10 \mathrm{~Hz}$ to $18 \mathrm{~Hz}$, and then to $24 \mathrm{~Hz}$ (Fig. 3), if previous change is unsuccessful. If this action fails, the next step is to change the target sensing threshold. The threshold should be increased from the default $25 \%$ gradually

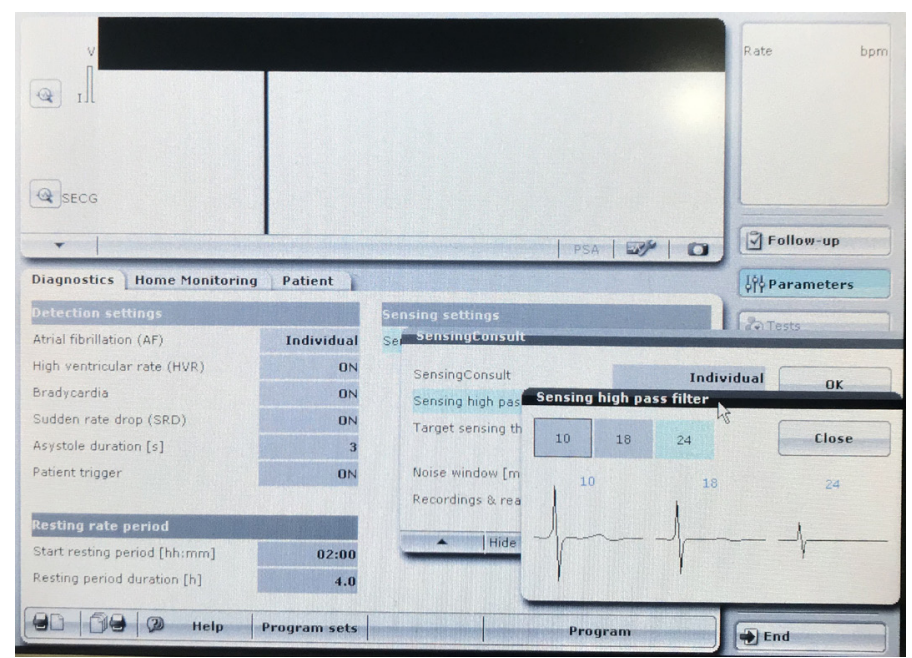

Figure 3.

up to $50 \%$ to succeed (Fig. 4). Naturally, we have not left the patient without any change in the device settings. Firstly, the sensing high pass filter was increased from the nominal

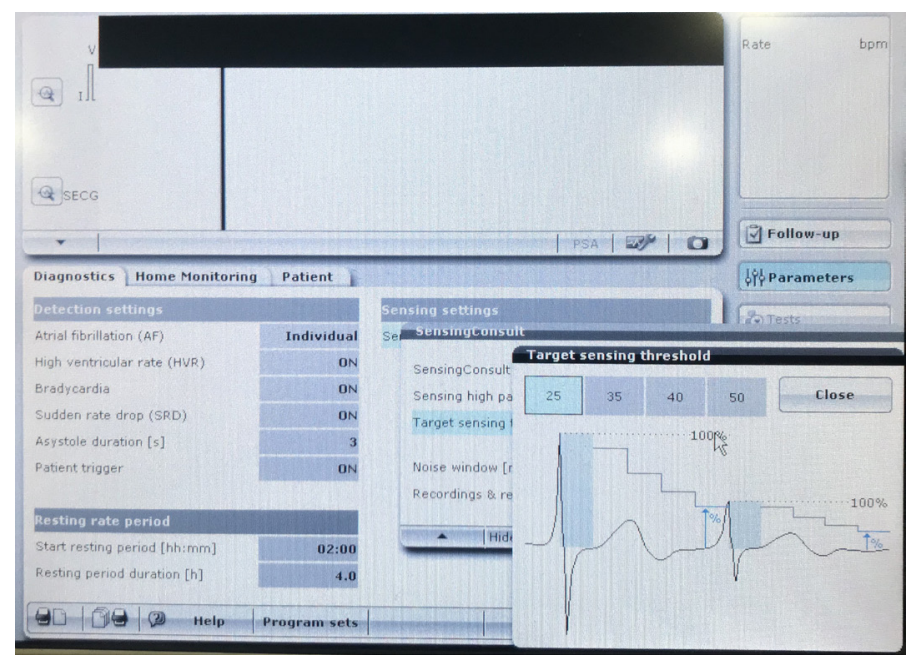

Figure 4.

value $10 \mathrm{~Hz}$ to $24 \mathrm{~Hz}$. Occurrence of oversensing episodes diminished significantly, but the problem continued to exist. Then, the target sensing threshold was increased to $50 \%$. The patient remains in our follow-up; we are awaiting the results of device reprogramming. Moreover, no plausible cause of the patient's syncope has been identified in the follow-up so far, so the patient still needs to be under the doctors' observation.

PWOS can be a frustrating problem, causing unnecessary reports of episodes of purported HVR, which turn out to be simple sinus rhythm. However, changing the device settings can be a successful way to stop oversensing. 


\section{References}

1. Lacour P, Long Dang P, Huemer M, Shokor Parwani A, Attanasio P, Pieske B, Boldt LH, Haverkamp $W$, Blaschke F. Performance of the new BioMonitor 2-AF insertable cardiac monitoring system: can better be worse?, Pacing Clin Electrophysiol, 2017;40(5):516526

2. Houmsse M, Ishola A, Daoud EG. Clinical utility of implantable loop recorders. Postgrad Med 2014; 126:30-37.

3. Moya A, Sutton R, Ammirati F, Blanc JJ, Brignole M, Dahm JB, Deharo JC, et al. Guidelines for the diagnosis and management of syncope (version 2009). Eur Heart J 2009; 30:2631-2671.

4. Drak-Hernandez Y, Toquero-Ramos J, Fernandez JM, Perez-Pereira E, Castro-Urda V, Fernandez-Lozano I. Effectiveness and safety of remote monitoring of patients with an implantable loop recorder. Rev Esp Cardiol (Engl Ed) 2013; 66:943-948. 